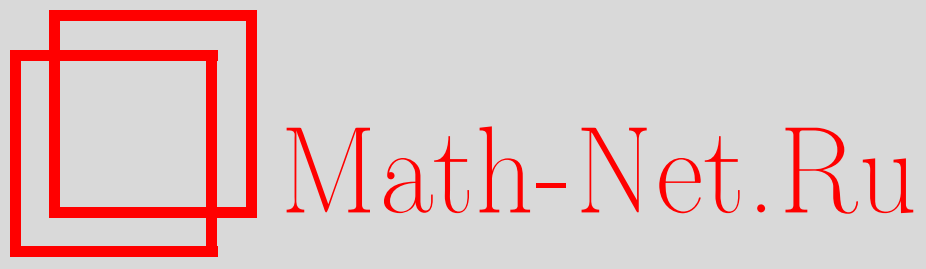

Г. М. Губреев, Регулярные ядра Миттаг-Леффлера и вольтерровы операторы, Функи. анализ и его прил., 2004, том 38, выпуск 4, 82-86

DOI: https://doi.org/10.4213/faa129

Использование Общероссийского математического портала MathNet.Ru подразумевает, что вы прочитали и согласны с пользовательским соглашением

http://www . mathnet.ru/rus/agreement

Параметры загрузки:

IP : 54.174 .149 .18

26 апреля 2023 г., 14:57:52

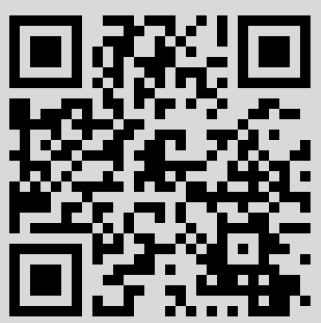


УДК $517.43+513.88$

\title{
Регулярные ядра Миттаг-Леффлера и вольтерровы операторы
}

\author{
(C) 2004. Г. М. ГУБРЕЕВ
}

1. Регулярные ядра Миттаг-Леффлера. В заметке изучается класс целых функций $\mathscr{E}_{\rho}: \mathbb{C} \rightarrow \mathfrak{H}$ порядка роста $\rho$ и нормального типа со значениями в сепарабельном гильбертовом пространстве $\mathfrak{H}$, который выделяется следующим определением.

ОПредЕЛЕНИЕ 1. Целую функцию $\mathscr{E}_{\rho}, \rho \geqslant 1$, назовем ядром Миттаг-Леффлера, если выполняются следующие условия:

1) для каждого $z \in \mathbb{C}$ существует такой вектор $h(z) \in \mathfrak{H}$, что для всех $\lambda \in \mathbb{C}$

$$
\left(\widetilde{\mathscr{E}}_{\rho}(\lambda), \mathscr{E}_{\rho}(z)\right)=\left(\mathscr{E}_{\rho}(\lambda), h(z)\right), \quad \text { где } \widetilde{\mathscr{E}}_{\rho}(\lambda):=\lambda^{-1}\left(\mathscr{E}_{\rho}(\lambda)-\mathscr{E}_{\rho}(0)\right) ;
$$

$2)$ для любых конечных наборов $\left\{c_{k}\right\}_{1}^{n},\left\{\lambda_{k}\right\}_{1}^{n}(n \geqslant 1)$ комплексных чисел значения форм $\left(\sum_{k=1}^{n} c_{k} \widetilde{\mathscr{E}}_{\rho}\left(\lambda_{k}\right), \sum_{k=1}^{n} c_{k} \mathscr{E}_{\rho}\left(\lambda_{k}\right)\right)$ принадлежат углу $0 \leqslant \arg z \leqslant \pi / \rho$.

Через $\Lambda^{\text {(exp) } о б о з н а ч а е т с я ~(с м . ~[1, ~ с . ~ 70]) ~ м н о ж е с т в о ~ о г р а н и ч е н н ы х ~ о п е р а т о р о в ~}$ $B$ в пространстве $\mathfrak{H}$, таких, что 1) $\sigma(B)=\{0\}, \operatorname{Ker} B=\{0\}, 2) \frac{1}{2 i}\left(B-B^{*}\right) \geqslant 0$, $3)$ оператор-функция $(I-z B)^{-1}$ имеет первый порядок роста и нормальный тип. Пусть $B^{1 / \rho}$ обозначает $\pi / \rho$-секториальную дробную степень диссипативного оператора $B$ (см. $\left[2\right.$, с. 195]), т. е. $\arg \left(B^{1 / \rho} h, h\right) \in[0, \pi / \rho], h \in \mathfrak{H}$. Тогда каждая целая функция

$$
\mathscr{E}_{\rho}(z):=\left(I-z B^{1 / \rho}\right)^{-1} g, \quad g \in \mathfrak{H}, B \in \Lambda^{(\exp )},
$$

имеет нормальный тип при порядке роста $\rho$ и является ядром Миттаг-Леффлера, поскольку

$$
\widetilde{\mathscr{E}}_{\rho}(z)=B^{1 / \rho}\left(I-z B^{1 / \rho}\right)^{-1} g, \quad\left(\sum c_{k} \widetilde{\mathscr{E}}_{\rho}\left(\lambda_{k}\right), \sum c_{k} \mathscr{E}_{\rho}\left(\lambda_{k}\right)\right)=\left(B^{1 / \rho} h, h\right),
$$

где $h=\sum c_{k} \mathscr{E}_{\rho}\left(\lambda_{k}\right)$. Отметим, что определение 1 пригодно лишь при $\rho \geqslant 1$. Поэтому для значений $\rho \in(0,1)$ ядром Mиттаг-Леффлера हீ будем называть произвольную вектор-функцию вида (1). В настоящей работе для значений параметра $\rho>1 / 2$ дается полное описание ядер Миттаг-Леффлера, которые обладают определенными свойствами регулярности.

ОПРЕДЕЛЕНИЕ 2 . Ядро $\mathscr{E}_{\rho}, \rho>1 / 2$, со значениями в пространстве $\mathfrak{H}$ называется непрерывно регулярным (в дальнейшем с-регулярным), если существуют такие константы $m, M(0<m<M<\infty)$, что для всех $h \in \mathfrak{H}$ справедливы оценки

$$
m\|h\|^{2} \leqslant \int_{\gamma_{\rho}}\left|z-\omega_{\rho}\right|^{\rho-1}\left\|\mathscr{E}_{\rho}(z)\right\|^{-2}\left|\left(\mathscr{E}_{\rho}(z), h\right)\right|^{2}|d z| \leqslant M\|h\|^{2}, \quad \omega_{\rho}:=e^{-i \pi /(2 \rho)},
$$

где $\gamma_{\rho}-$ угол с вершиной $z=0$ и сторонами $\arg z=0, \arg z=-\pi / \rho$.

Обозначим через $\gamma_{\rho}^{-}$область внутри $\gamma_{\rho}$, т. е. $\gamma_{\rho}^{-}:=\{z \in \mathbb{C}:-\pi / \rho<\arg z<0\}$. Пусть $\lambda^{\rho}$ - та ветвь многозначной функции, которая отображает $\gamma_{\rho}^{-}$на нижнюю полуплоскость $\mathbb{C}_{-}$. Последовательность $\Lambda:=\left\{\lambda_{k}\right\}_{-\infty}^{+\infty}, \lambda_{k} \in \gamma_{\rho}^{-}$, лежащую в некоторой криволинейной полосе $-\Delta<\operatorname{Im} \lambda^{\rho}<-\delta(\delta, \Delta>0)$ и сгущающую- 
ся к $\infty$, назовем последовательностью без пропусков, если для любого $x \in \mathbb{R}$ существует такой элемент $\lambda(x) \in \Lambda$, что $\sup _{x \in \mathbb{R}}\left|\lambda^{\rho}(x)-x\right|<\infty$.

ОпредЕЛЕниЕ 3. Ядро Миттаг-Леффлера $\mathscr{E}_{\rho}, \rho>1 / 2$, со значениями в пространстве $\mathfrak{H}$ называется дискретно регулярным (в дальнейшем $d$-регулярным), если существует хотя бы одна последовательность без пропусков $\Lambda$, для которой семейство $\left\{\mathscr{E}_{\rho}\left(\lambda_{k}\right): \lambda_{k} \in \Lambda\right\}$ образует безусловный базис пространства $\mathfrak{H}$.

ОПРЕДЕЛЕНИЕ 4. Ядро $\mathscr{\varrho}$ н называется регулярным, если оно одновременно $c$-регулярно и $d$-регулярно.

Следуя работам [3, 4], приведем теперь одну конструкцию регулярных ядер со значениями в каждом из пространств $L_{2}(0, \sigma), \sigma>0$. Пусть на контуре $\gamma_{\rho}, \rho>$ $1 / 2$, который фигурирует в определении 2 , задан $A_{\gamma_{\rho}}^{2}$-вес Макенхаупта $w^{2}$, т. е.

$$
\sup _{z \in \gamma_{\rho}} \sup _{r>0}\left\{r^{-1} \int_{B(z, r) \cap \gamma_{\rho}} w^{2}(\lambda)|d \lambda| r^{-1} \int_{B(z, r) \cap \gamma_{\rho}} w^{-2}(\lambda)|d \lambda|\right\}<\infty,
$$

где $B(z, r)$ - круг с центром $z$ и радиуса $r$. Тогда существует аналитическая в угле $\gamma_{\rho}^{-}$функция $w_{-}$, удовлетворяющая следующим условиям: а) почти при всех $z \in \gamma_{\rho}$ существуют некасательные предельные значения $w_{-}(z):=$ $\lim _{\lambda \rightarrow z} w_{-}(\lambda), \lambda \in \gamma_{\rho}^{-}$, причем $\left.w^{2}(z) \stackrel{\text { п.в }}{=}\left|w_{-}(z)\right|^{2}, z \in \gamma_{\rho} ; \mathrm{b}\right)$ функция $w_{-}\left(u^{1 / \rho}\right)$ - внешняя в нижней полуплоскости $\mathbb{C}_{-}$(определение см. в [5, с. 73]). Этими условиями $w_{-}$определяется с точностью до унимодулярного постоянного множителя. Имеет место интегральное представление

$$
w_{-}\left(u^{1 / \rho}\right)=u^{(1+\rho) /(2 \rho)} \int_{0}^{\infty} e^{-i t u} y_{w}(t) d t, \quad u \in \mathbb{C}_{-},
$$

с некоторой функцией $y_{w} \in L_{2}^{\text {loc }}\left(\mathbb{R}_{+}\right)$. Через $y_{w}^{\sigma}$ обозначим сужение функции $y_{w}$ на отрезок $[0, \sigma]$. Итак, каждый $A_{\gamma_{\rho}}^{2}$-вес $w^{2}$, в полном соответствии с формулой (1) в частном случае $B=J_{\sigma} \in \Lambda^{(\mathrm{exp})}, g=y_{w}^{\sigma}, \mathfrak{H}=L_{2}(0, \sigma)$, порождает ядро

$$
\mathscr{E}_{\rho, w}(z, t):=\left(I-z J_{\sigma}^{1 / \rho}\right)^{-1} y_{w}^{\sigma}, \quad\left(J_{\sigma}^{1 / \rho} h\right)(t)=e^{i \pi /(2 \rho)} \Gamma^{-1}\left(\frac{1}{\rho}\right) \int_{0}^{t}(t-s)^{1 / \rho-1} h(s) d s,
$$

со значениями в пространстве $L_{2}(0, \sigma)$. В дальнейшем ядра $\mathscr{E}_{\rho, w}(z, t)$ будем называть $ш$-ядрами Миттаг-Леффлера.

ТЕОремА 1. Для произвольного $A_{\gamma_{\rho}}^{2}$-веса $w^{2}$ ядро $\mathscr{E}_{\rho, w}(z, t), \rho>1 / 2$, со значениями в каждом из пространств $L_{2}(0, \sigma), \sigma>0$, является регулярным.

Если в формулах (3) отправляться от степенного веса $w^{2}(z)=|z|^{\omega},-1<$ $\omega<1$, то нетрудно проверить [3], что соответствующее $w$-ядро выражается через функцию Миттаг-Леффлера $E_{\rho}(z, \mu)$ по формуле

$$
\mathscr{E}_{\rho, w}(z, t)=t^{\mu-1} E_{\rho}\left(e^{i \pi /(2 \rho)} z t^{1 / \rho} ; \mu\right), \quad E_{\rho}(z ; \mu):=\sum_{k=0}^{\infty} \Gamma^{-1}\left(\mu+k \rho^{-1}\right) z^{k},
$$

где $\mu=(2 \rho)^{-1}(1+\rho-\omega)$. Этим объясняется то, что абстрактные классы функций $\mathscr{E}_{\rho}$, которые выделяются определением 1 , были названы нами ядрами Миттаг-Леффлера. Функция Миттаг-Леффлера и ее обобщения занимают заметное место в современном анализе [7-11]. Свойства интегральных преобразований с ядрами Миттаг-Леффлера вида (4) составляют одну из основных тем монографии [6]. М. Г. Крейн неоднократно указывал на необходимость переосмыслить ряд результатов этой книги, полученных М. М. Джрбашяном 
классическими методами, с позиций бурно развивающейся теории несамосопряженных операторов. В беседе с автором (1986 г.) он предложил выяснить, насколько существенна роль операторов дробного интегрирования в построениях Джрбашяна и не порождают ли другие вольтерровы операторы интегральные преобразования с аналогичными свойствами. В принятой здесь терминологии эту задачу можно сформулировать как задачу об описании всех $c$-регулярных ядер Миттаг-Леффлера.

ОПРЕДЕЛЕНИЕ 5. Ядра $\mathscr{E}_{\rho}^{(k)}$ со значениями в гильбертовых пространствах $\mathfrak{H}_{k}(k=1,2)$ называются изоморфнымми, если существует непрерывный и непрерывно обратимый оператор $S$ из $\mathfrak{H}_{1}$ на $\mathfrak{H}_{2}$, такой, что $S \mathscr{E}_{\rho}^{(1)}(z)=\mathscr{E}_{\rho}^{(2)}(z), z \in \mathbb{C}$.

Очевидно, что изоморфные ядра только одновременно могут обладать свойством какой-нибудь регулярности. Поэтому описание регулярных ядер МиттагЛеффлера возможно лишь с точностью до изоморфизма.

2. Описание регулярных ядер Миттаг-Леффлера. Если ядро $\mathscr{E}_{\rho}$ имеет нормальный тип $\sigma$ при порядке роста $\rho$, то будем говорить, что его рост $p a-$ вен $(\rho, \sigma)$. Следующий результат показывает, что регулярные ядра надо искать среди вектор-функций вида (1).

ТЕОрема 2. Каждое с-регулярное или d-регулярное ядро Миттаг-Лефблера $\mathscr{E}_{\rho}$ роста $(\rho, \sigma), \rho \geqslant 1$, со значениями в сепарабельном гильбертовом пространстве $\mathfrak{H}$ допускает единственное представление вида

$$
\mathscr{E}_{\rho}(z)=\left(I-z B^{1 / \rho}\right)^{-1} g, \quad g \in \mathfrak{H}, B \in \Lambda^{(\exp )},
$$

причем экспоненциальный тип оператора $(I-z B)^{-1}$ равен $\sigma$.

Из теоремы 1 следует, что регулярными будут все изоморфные образы $w$-ядер, построенных с помощью канонической процедуры по произвольному $A_{\gamma_{\rho}}^{2}$-весу $w^{2}$ на контуре $\gamma_{\rho}$. Оказывается, что других регулярных ( $c$-регулярных, $d$-регулярных) ядер Миттаг-Леффлера нет.

ТЕОРема 3. Пусть $\mathscr{E}_{\rho}-$ произвольное ядро Миттаг-Леффлера, $\rho>1 / 2$, роста $(\rho, \sigma)$ со значениями в сепарабельном гильбертовом пространстве $\mathfrak{H}$. Тогда справедливы следуюшие утверждения:

1) ядро $\mathscr{E}_{\rho}$ с-регулярно тогда и только тогда, когда оно d-регулярно;

2) ядро $\mathscr{E} \rho$ регулярно тогда и только тогда, когда вес

$$
w^{2}(z):=\left|z-\omega_{\rho}\right|^{1-\rho}\left\|\mathscr{E}_{\rho}(z)\right\|^{2}
$$

удовлетворяет условию $\left(A_{\gamma_{\rho}}^{2}\right)$ и $\mathscr{E}_{\rho}$ изоморфно ядру $\mathscr{E}_{\rho, w}$ со значениями в пространстве $L_{2}(0, \sigma)$, построенному по весу $w^{2}$.

В сформулированной теореме речь идет о случае $\rho>1 / 2$. Построения этой работы непригодны для значений параметра $\rho \in(0,1 / 2)$, что объясняется, в основном, неограниченным ростом резольвенты $\left(I-z B^{1 / \rho}\right)^{-1}, B \in \Lambda^{(\exp )}$, вдоль любого луча $\arg z=\varphi$. Поэтому при $0<\rho<1 / 2$ правильные постановки задач должны быть другими. В пограничном случае $\rho=1 / 2$ имеют место аналогичные утверждения, но с несколько другими формулировками. Эти результаты опубликованы в [14]. Наиболее простой случай $\rho=1$ впервые исследовался в работах $[12,13]$.

3. Некоторые приложения. $\mathrm{K}$ понятию регулярного ядра Миттаг-Леффлера мы приходим при изучении спектральной структуры конечномерных возмущений дробных степеней операторов класса $\Lambda^{(\exp )}$. Например, к этому 
понятию приводит задача о подобии нормальному оператору произвольного одномерного возмущения вида

$$
K h:=B^{1 / \rho} h+(h, f) g, \quad B \in \Lambda^{(\exp )}, f, g \in \mathfrak{H}, \rho>1 / 2 .
$$

Фредгольмов спектр оператора $K$, т. е. множество $\left\{\mu^{-1}: \mu \in \sigma(K), \mu \neq 0\right\}$, совпадает с множеством корней целой функции $\Phi(z):=1-z\left(\left(I-z B^{1 / \rho}\right)^{-1} g, f\right)$, которое мы обозначим через $\Lambda$. Скажем, что последовательность $\Lambda$ расположена правильно относительно контура $\gamma_{\rho}$, если те ее части, которые при некотором $\varepsilon>0$ попали в углы $|\arg z|<\varepsilon,|\pi / \rho+\arg z|<\varepsilon$, удовлетворяют условию $\left|\operatorname{Im} \lambda_{k}^{\rho}\right| \geqslant \delta>0$, где $\lambda^{\rho}$ - аналитическое продолжение ветви, отображающей $\gamma_{\rho}^{-}$ на $\mathbb{C}_{-}$. Если $\Lambda$ расположена правильно $(0 \notin \Lambda)$, то $\Lambda_{ \pm}=\Lambda \cap \gamma_{\rho}^{ \pm}$, где $\gamma_{\rho}^{+}$- дополнительный угол, т. е. $\gamma_{\rho}^{+}:=\{z \in \mathbb{C}: 0<\arg z<\pi / \alpha\}, \rho^{-1}+\alpha^{-1}=2$. Поэтому обе последовательности $U:=\left\{u_{k}\right\}, V:=\left\{v_{k}\right\}, u_{k}:=\lambda_{k}^{\alpha}, \lambda_{k} \in \Lambda_{+}, v_{k}:=\overline{\lambda_{k}^{\rho}}$, $\lambda_{k} \in \Lambda_{-}, \alpha^{-1}+\rho^{-1}=2$, лежат в $\mathbb{C}_{+}$. Напомним, что последовательность из верхней полуплоскости (например, $U$ ) удовлетворяет условию Карлесона, если

$$
\inf _{k} \prod_{j \neq k} \frac{\left|u_{k}-u_{j}\right|}{\left|u_{k}-\bar{u}_{j}\right|}>0, \quad U=\left\{u_{k}\right\} .
$$

ТЕОрема 4. Если $K$ - произволъный оператор вида (5), то справедливы следующие утверждения:

а) Пусть фредгольмов спектр оператора $K$ есть некоторая последовательность без пропусков. Если $K$ подобен нормальному оператору, то ядро $\mathscr{E}_{\rho}(z):=$ $\left(I-z B^{1 / \rho}\right)^{-1} g$ регулярно.

b) Пусть указанное ядро $\mathscr{E}_{\rho}$ регулярно и имеет рост $(\rho, \sigma), w^{2}(z):=$ $\left|z-\omega_{\rho}\right|^{1-\rho}\left\|\mathscr{E}_{\rho}(z)\right\|^{2}$ и корни функиии $\Phi$ расположены правилъно относительно угла $\gamma_{\rho}$. Тогда для подобия оператора $K$ нормальному оператору необходимо $u$ достаточно выполнение следующих условий:

1) множество корней $\Phi$ бесконечно и все они простые;

2) $\limsup _{r \rightarrow \infty} r^{-\rho} \log \left|\Phi\left(r e^{-\pi /(2 \rho)}\right)\right|=\sigma, \limsup _{r \rightarrow \infty} r^{-\alpha} \log \left|\Phi\left(r e^{-i \pi /(2 \alpha)}\right)\right|=0$, $\alpha^{-1}+\rho^{-1}=2$;

3) вес $W^{2}(z):=w^{-2}(z)|\Phi(z)|^{2}$ удовлетворяет условию $\left(A_{\gamma_{\rho}}^{2}\right)$;

4) последовательности $U, V$ удовлетворяют условию $(\mathrm{C})$.

Для конкретных приложений необходимо иметь простые признаки регулярности ядер Миттаг-Леффлера. Сформулируем здесь один из результатов такого рода. Пусть $B \in \Lambda^{(\text {ехр)}}$, причем $\operatorname{Im} B$ ядерна. Пусть также существуют число $a \in \mathbb{C}$ и векторы $f, f_{1} \in B \mathfrak{H}+B^{*} \mathfrak{H}$, такие, что $F(z):=a-z\left((I-z B)^{-1} f, f_{1}\right)$ является функцией типа синуса [15], ширина индикаторной диаграммы которой равна $2 \operatorname{SpIm} B$. Эти условия обеспечивают принадлежность $f$ к области определения оператора $B^{\mu-1}, \mu>1 / 2$. Доказывается, что ядро Миттаг-Лефбблера $\mathscr{E}_{\rho, \mu}(z):=\left(I-z B^{1 / \rho}\right)^{-1} B^{\mu-1} f, \mu>1 / 2$, регулярно тогда и только тогда, когда $1 / 2<\mu<1 / 2+1 / \rho$. Более того, регулярное ядро हீ, значениями в пространстве $L_{2}(0, \sigma), \sigma:=2 \operatorname{SpIm} B$.

\section{ЛитЕРАТУРА}

1. Бродский M. C. Треугольные и жордановы представления линейных операторов. Наука, М., 1969. 2. Секефалъви-Надъ Б., Фойаш Ч. Гармонический анализ операторов в гильбертовом пространстве. Мир, М., 1970. 3. Губреев Г. М. Записки научных семинаров ЛОМИ. ХІХ, 190, 34-80 (1991). 4. Губреев Г. М. Спектральный анализ биортогональных разложений, порождаемых весами Макенхаупта. Дисс. д.ф.-м.н., 
Харьков, ФТИНТ, 1995. 5. Гарнетm Дж. Ограниченные аналитические функции. Наука, М., 1984. 6. Джрбашян M. M. Интегральные преобразования и представления функций в комплексной области. Наука, М., 1966. 7. Ostrovskii I. V., Peresyolkova I. N. In: Theory of Functions and Applications. Collection of works dedicated to the memory of M. M. Djrbashian, 1995, pp. 49-152. 8. Sedletskii A. M. Anal. Math. 20, 117-132 (1994). 9. Гельфанд И. М., Граев М. И., Ретах В. С. УМН, 53, вып. 1 (319), 3-60 (1998). 10. Маергойз Л. С. Алгебра и анализ, 12, вып. 2, 1-63 (2000). 11. Маергойз Л. С. Индикаторная и сопряженная диаграммы целой функции уточненного порядка. Препринт 789Ф, Красноярск, 1998. 12. Губреев Г. М. Матем. сб., 190, вып. 12, 3-36 (1999). 13. Губреев Г. М. Алгебра и анализ, 12, вып. 6, 1-97 (2000). 14. Губреев Г. М., Волкова М. Г. Зап. научн. семин. ПОМИ, 290, 23-41 (2002). 15. Левин Б. Я. Записки физ.-мат. ф-та ХГУ и ХМО, 27, 39-48 (1961).

Южноукраинский педагогический государственный университет (г. Одесса)

email: varv@rambler.ru

Поступило в редакцию 20 февраля 2003 г.

\title{
УДК 517.956
}

\section{Об усреднении периодических параболических систем*}

\author{
(с) 2004. Т. А. СуслинА
}

1. Рассматривается вопрос об усреднении (гомогенизации) задачи Коши для параболической системы вида

$$
\rho\left(\varepsilon^{-1} \mathbf{x}\right) \frac{\partial \mathbf{u}_{\varepsilon}(\mathbf{x}, t)}{\partial t}=-\widehat{\mathscr{A}}_{\varepsilon} \mathbf{u}_{\varepsilon}(\mathbf{x}, t), \quad \rho\left(\varepsilon^{-1} \mathbf{x}\right) \mathbf{u}_{\varepsilon}(\mathbf{x}, 0)=\boldsymbol{\phi}(\mathbf{x}),
$$

в пределе малого периода $\varepsilon$, стремящегося к нулю. Здесь $\mathbf{u}_{\varepsilon}(\mathbf{x}, t)$ есть $\mathbb{C}^{n}$-значная функция от $\mathbf{x} \in \mathbb{R}^{d}$ и $t \geqslant 0, \widehat{\mathscr{A}}_{\varepsilon}-$ матричный эллиптический оператор с периодическими коэффициентами, зависящими от $\varepsilon^{-1} \mathbf{x}$, и $\rho-$ периодическая положительная матрица. Точное описание оператора $\widehat{\mathscr{A}}_{\varepsilon}$ см. ниже в разд. $2,4$. Задача гомогенизации для параболических уравнений активно изучалась традиционными методами теории усреднений (см., например, книги [1-3]). Соответствующие результаты дают сходимость (в подходящем функциональном классе) решений $\mathbf{u}_{\varepsilon}$ к решению $\mathbf{u}_{0}$ усредненной системы с постоянными эффективными коэффициентами. Мы используем абстрактный теоретико-операторный подход, развитый применительно к эллиптическим системам в статьях $[4,5]$. Этот подход позволяет не только установить сходимость $\mathbf{u}_{\varepsilon}$ к $\mathbf{u}_{0}$ по норме в $L_{2}\left(\mathbb{R}^{d}\right)$ (при фиксированном $t$ ), но и получить оценку для нормз разности $\mathbf{u}_{\varepsilon}-\mathbf{u}_{0}$, точную по порядку и равномерную относительно $L_{2}\left(\mathbb{R}^{d}\right)$-нормъ начального значения $\phi$. Точнее говоря, получается оценка порядка $\varepsilon t^{-1 / 2}$ для операторной нормы разности разрешающих операторов для задачи (1) и для «усредненной» задачи Коши (см. ниже теоремы 3,4$)$.

Пусть $\mathfrak{H}$ - сепарабельное гильбертово пространство. Символ $\|\cdot\|_{\mathfrak{H}}$ означает норму в $\mathfrak{H} ;$ символ $\|\cdot\|_{\mathfrak{H} \rightarrow \mathfrak{H}}$ означает норму ограниченного оператора в $\mathfrak{H}$. Далее, $|\cdot|$ - норма вектора в $\mathbb{C}^{n}$, а $\mathbf{1}_{n}-$ единичная $(n \times n)$-матрица. Используем обозначения $\mathbf{x}=\left(x^{1}, \ldots, x^{d}\right) \in \mathbb{R}^{d}, i D_{j}=\partial / \partial x^{j}, j=1, \ldots, d$,

*Работа выполнена при поддержке РФФИ (грант 02-01-00798). 